\title{
Smart Industries in Industry4.0: An Iot and Cloud Based Real Time Energy Meter Monitoring System
}

\author{
Prateek Agrawal, Himmat Khatik, Yogendra Singh Solanki, Aditya Maheshwari, Vivek Jain
}

\begin{abstract}
With increase in low cost internet and with advent of $4 G$ technologies increase in internet speed, IoT-based applications are getting more popular day by day and it provides effective solution of many real time monitoring problems. In this research, a cloud based real-time monitoring system for industrial energy meter has been proposed. The prototype system provides continuous and ubiquitous access to energy consumption of the equipment under monitoring to the consumer using IoT technology. To implement the system in any industry it requires a simple and low-cost upgrade to the existing meters rather than complete replacement. Based on the experimental analysis, it is found that from the collected data, it is possible to obtain the pattern of consumption as well as faultiness present in the existing system. In terms of future scope of work, the presented work can also be extended to grid distribution level from which load distributed in the area can be estimated so that the system can be strengthened to enhance performance.
\end{abstract}

Keywords: - CRC: Cyclical Redundancy Check, LRC: Longitudinal Redundancy Check, THD: Total Harmonic Distortions: Universal Serial Bus, Smart Energy meter, IoT, Cloud, and Load optimization.

\section{INTRODUCTION}

E nergy management is becoming crucial day by day. Today, energy costs have become one of the major factors in industrial as well as domestic budgeting, wherefore machine power consumption monitoring is important for most of modern industries.

Most of the production plants, organizations, and factories face several challenges in their day to day plant operation and the biggest one is the energy consumption monitoring, due to their massive scale and diversified infrastructure, lack of readings or actionable data related to their energy consumption.

For industries real time insights of machine's availability, its power consumption and performance is a very crucial part to run the business smoothly, with real time cloud based monitoring of equipment's downtimes, it empowers the owner or the manager to reduce undesired and sudden equipment breakdowns, to get the maximum output by utilizing the available machinery in optimized manner.

\section{Revised Manuscript Received on March 15, 2020.}

Prateek Agrawal, Department of Electronics and Communication Engineering, Techno India NJR Institute of Technology, College, Biliya, Rajasthan, India.

Himmat Khatik, Department of Electronics and Communication Engineering, Techno India NJR Institute of Technology, College, Biliya, Rajasthan, India.

Yogendra Singh Solanki, Asst. Professor, Department of Electronics and Communications, Techno India NJR Institute of Technology, Udaipur, India.

Aditya Maheshwari, Capacity of Project Lead- New Initiative \& Research, Techno India NJR Institute of Technology Udaipur, India.

Dr. Vivek Jain, Assistant Professor, Techno India NJR Institute of Technology, Udaipur, India.
The proposed System fetches the real-time energy consumption readings from the meter installed on the equipment and provides the machine on-time, off-time, efficiency, and power consumption details on smart phone or Personal Computer. Unlike traditional energy management systems, the proposed real time energy consumption monitoring system provides a convenient way to accumulate and analyse power quality.

\section{Benefits of Power Monitoring Solutions:}

- Abnormality detection \& Reduction in Energy Consumption

- Cost Optimization

- Real-time Energy Insights

- Increased Performance of the available Facility

- Upcoming Energy Needs prediction

- Reduced Unnecessary Equipment running

Our Low-cost monitoring system collects the following parameters from panel meter mounted on all the machineries and send to the microprocessor/ microcontroller which upload all the data to the cloud dashboard which helps to monitor the data anywhere in the world.

The objective of this research project is to develop an IoTbased energy monitoring system for the industrial sector. The proposed system is integration of the advanced low cost SoC (system on Chip) based single board computers and cloud services. The rest of the paper is organized as follows. Section II provides an overview of the system. Section III describes the working of the proposed system. Section IV discusses the results and Section V concludes the paper with future work.

\section{SYSTEM OVERVIEW}

The System block diagram as shown in the figure. It consists of different part as Raspberry Pi-3 Model B+, USB to RS485 Converter and Meter (elite-440).

\section{A. Raspberry Pi}

In this System Raspberry Pi is Main part of the system. The Raspberry Pi is a very low-cost pocket size, single board computer that can plugs into monitor and also Connect with the Mouse and Keyboard through USB port. The Raspberry $\mathrm{Pi}$ has $4 \times \mathrm{ARM}$ Cortex-A53 of $1.2 \mathrm{GHz} \mathrm{CPU}$ and $1 \mathrm{~GB}$ LPDDR2 RAM @ 900MHz and It has $2.4 \mathrm{GHz} 802.11 \mathrm{n}$ wireless Wi-Fi or $4 \times$ USB 2.0, Ethernet port.

\section{B. Elite 440 Panel meter}

The Elite 440 is a three-phase digital panel meter for accurate and reliable measurement of electrical parameters such as voltage, 
current, power, frequency so on for industrial and home applications. The elite 440 can easily interface with external device as it is Modus [Rs-485] enable. It can be used for both star or delta connections and low or high voltage applications measurement. It has high accuracy of Class $0.2 \mathrm{~s}, 0.5 \mathrm{~s}, 0.1$. It also provides Average THD measurement for voltage, current and power.

\section{USB to RS-485 converter Module}

The RS-485 was developed for High speed and max data rate in minimum time's to RS-485 converter is used to perform USB to two wire RS-485 (Half Duplex) and vice versa conversion. Module can power up by the USB bus. RS-485 has maximum transfer data rate of $10 \mathrm{Mbps}$ at maximum distance of about 50 feet.

\section{SYSTEM OPERATION}

Elite 440 panel meter is used to measure several electrical parameters and supported Rs-485 Modbus Protocol, which is mounted on the 3-phase Motor. A Raspberry Pi is used to continuously capture the value of parameter via Modbus protocol from the elite 440 and upload it to cloud. Basic Block Diagram of Low-Cost Monitoring System is shown in figure 2. The Raspberry pi is act as a master and elite 440 as a slave device for Modbus protocol, which is connected through a USB-RS485 converter.

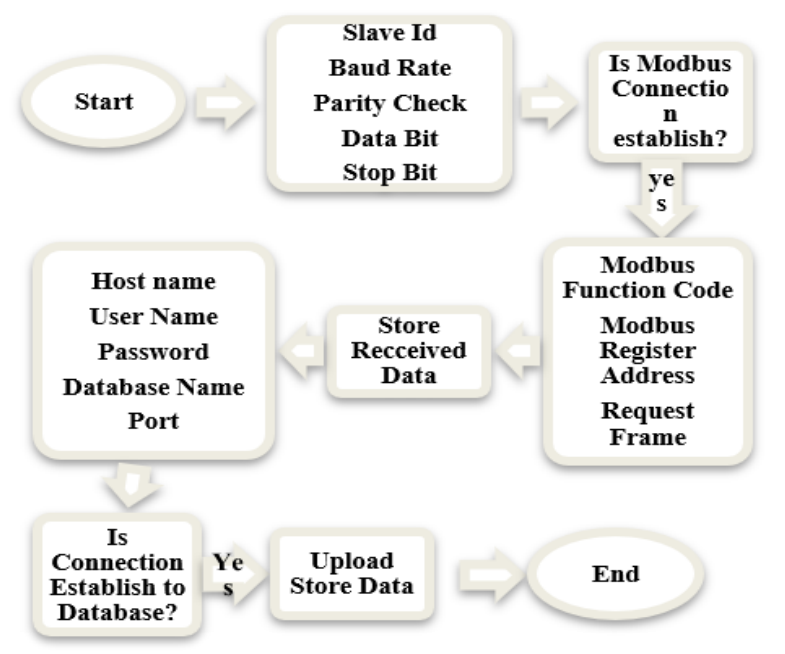

Fig. 1: Flow Chart

Modbus is a serial communication protocol works as master/slave communication. It enables communication among many devices connected to the same network. In one network there is one master and 246 slave devices communicate to each other. Each device communicating on a Modbus is given unique address. Each slave has different register to store several values in it, which can be access through Modbus communication by using Modbus function codes the value at registers can be read/write depends on type of register. Every communication has been checked by CRC/LRC. Flowchart of Low-Cost Monitoring System is shown in figure 1 .



Fig. 2. Block Diagram
During Initialization, serial communication between raspberry pi and elite 440 is establish by providing the correct slave information such as slave id, baud rate, parity check, data bit, stop bit. After the successful connection raspberry pi send read register request to elite 440 with Modbus register address given is table 1 elite 440 calculate CRC of receive message frame then compare it to the received CRC if CRC matches then elite 440 respond back to master request elite 440 sends the store data of register to raspberry pi and then raspberry pi upload the data to the cloud.

\begin{tabular}{|c|c|c|c|c|c|}
\hline Start & Slave Id & Function & Modbus & CRC & End \\
3.5 char & 8-bit & $\begin{array}{c}\text { Code } \\
\text { 8-bit }\end{array}$ & $\begin{array}{c}\text { Register } \\
\text { Address } \\
\text { 16-bit }\end{array}$ & 16-bit & 3.5 char \\
& & & \\
\hline
\end{tabular}

Fig.3. Modbus Request Message Frame

Table 1: elite 440 Modbus register address

\begin{tabular}{|c|c|c|c|c|}
\hline Sr. No. & $\begin{array}{l}\text { MODBUS } \\
\text { Register }\end{array}$ & Parameter & Unit & $\begin{array}{l}\text { In } \\
\text { Hex }\end{array}$ \\
\hline 1 & 40100 & $\begin{array}{l}\text { R Phase to Neutral } \\
\text { Voltage }\end{array}$ & Volt & $0 x 63$ \\
\hline 2 & 40102 & $\begin{array}{l}\text { Y Phase to Neutral } \\
\text { Voltage }\end{array}$ & Volt & $0 x 65$ \\
\hline 3 & 40104 & $\begin{array}{l}\text { B Phase to Neutral } \\
\text { Voltage }\end{array}$ & Volt & $0 x 67$ \\
\hline 4 & 40106 & Average Voltage & Volt & $0 \times 69$ \\
\hline 5 & 40108 & RY Voltage & Volt & 0x6B \\
\hline 6 & 40110 & YB Voltage & Volt & $0 \times 6 \mathrm{D}$ \\
\hline 7 & 40112 & BR Voltage & Volt & $0 \mathrm{X} 6 \mathrm{~F}$ \\
\hline 8 & 40114 & R Phase Line current & Ampere & $0 \times 71$ \\
\hline 9 & 40116 & Y Phase Line current & Ampere & $0 \times 73$ \\
\hline 10 & 40118 & B Phase Line current & Ampere & $0 \times 75$ \\
\hline 11 & 40120 & Neutral Line current & Ampere & $0 \times 77$ \\
\hline 12 & 40122 & R Phase Active Current & Ampere & $0 \times 79$ \\
\hline 13 & 40124 & Y Phase Active Current & Ampere & 0X7B \\
\hline 14 & 40126 & B Phase Active Current & Ampere & $0 \times 7 \mathrm{D}$ \\
\hline 15 & 40128 & R Phase Reactive Current & Ampere & $0 \mathrm{X} 7 \mathrm{~F}$ \\
\hline 16 & 40130 & Y Phase Reactive Current & Ampere & $0 \times 81$ \\
\hline 17 & 40132 & B Phase Reactive Current & Ampere & $0 \times 83$ \\
\hline 18 & 40134 & Power factor & & $0 \times 85$ \\
\hline 19 & 40136 & Power factor & & $0 \times 87$ \\
\hline 20 & 40138 & Power factor & & $0 \times 89$ \\
\hline 21 & 40140 & Average power factor & & 0X8B \\
\hline 22 & 40142 & R- Phase Active Power & $\mathrm{kW}$ & $0 \times 8 \mathrm{D}$ \\
\hline 23 & 40144 & Y-Phase Active Power & $\mathrm{kW}$ & $0 \times 8 \mathrm{~F}$ \\
\hline 24 & 40146 & B- Phase Active Power & $\mathrm{kW}$ & $0 \times 91$ \\
\hline 25 & 40148 & 3 Phase Active Power & KW & $0 \times 93$ \\
\hline 26 & 40150 & R- Phase Reactive Power & $\mathrm{kVAr}$ & $0 \times 95$ \\
\hline 27 & 40152 & Y- Phase Reactive Power & $\mathrm{kVAr}$ & $0 \times 97$ \\
\hline 28 & 40154 & B- Phase Reactive Power & $\mathrm{kVAr}$ & 0x99 \\
\hline 29 & 40156 & 3 Phase Reactive Power & $\mathrm{kVAr}$ & 0x9B \\
\hline 30 & 40158 & R- Phase Apparent Power & $\mathrm{kVA}$ & 0x9D \\
\hline 31 & 40160 & $\begin{array}{l}\text { Y- Phase Apparent } \\
\text { Power }\end{array}$ & $\mathrm{kVA}$ & $0 \times 9 F$ \\
\hline 32 & 40162 & B- Phase Apparent Power & $\mathrm{kVA}$ & 0xA1 \\
\hline 33 & 40164 & 3 Phase Apparent Power & $\mathrm{kVA}$ & $0 \mathrm{xA3}$ \\
\hline 34 & 40172 & Frequency $\mathrm{Hz}$ & $\mathrm{Hz}$ & $0 x A B$ \\
\hline 35 & 40174 & Meter real Time & Seconds & 0xAD \\
\hline 36 & 40178 & $\begin{array}{l}\text { R-Phase Voltage Total } \\
\text { Harmonic Distortion }\end{array}$ & $\%$ & 0XB 1 \\
\hline 37 & 40180 & $\begin{array}{l}\text { Y-Phase Voltage Total } \\
\text { Harmonic Distortion }\end{array}$ & $\%$ & 0XB3 \\
\hline 38 & 40182 & $\begin{array}{l}\text { B-Phase Voltage Total } \\
\text { Harmonic Distortion }\end{array}$ & $\%$ & 0xB5 \\
\hline 39 & 40184 & $\begin{array}{l}\text { R-Phase Current Total } \\
\text { Harmonic Distortion }\end{array}$ & $\%$ & 0xB7 \\
\hline 40 & 40186 & $\begin{array}{l}\text { Y-Phase Current Total } \\
\text { Harmonic Distortion }\end{array}$ & $\%$ & 0xB9 \\
\hline 41 & 40188 & $\begin{array}{l}\text { B-Phase Current Total } \\
\text { Harmonic Distortion }\end{array}$ & $\%$ & 0xBB \\
\hline 42 & 40190 & $\begin{array}{l}\text { R-Phase Power Total } \\
\text { Harmonic Distortion }\end{array}$ & $\%$ & $0 \mathrm{XBD}$ \\
\hline
\end{tabular}




\begin{tabular}{|l|l|l|l|l|}
43 & 40192 & $\begin{array}{l}\text { Y-Phase Power Total } \\
\text { Harmonic Distortion }\end{array}$ & $\%$ & 0xBF \\
\hline 44 & 40194 & $\begin{array}{l}\text { B-Phase Power Total } \\
\text { Harmonic Distortion }\end{array}$ & $\%$ & 0xC1 \\
\hline 45 & 40200 & Active Total - Import & $\mathrm{kWh}$ & 0xC9 \\
\hline
\end{tabular}

\section{RESULT}

The prototype of system is constructed as shown in fig.4 the prototype is presently installed in our college premises to monitor the real time power consumption. The system is connected to a three-phase water pump and on interval of 30 minutes the system fetches the meter readings and uploads the data to the cloud database.



Fig.4. System Setup

The meter readings of all the parameters which are shown in the table 1 are stored in the cloud database along with the time stamp. The consolidated data can be visualized on the cloud as chart, dashboard, graph and tablefig.5, fig.7, fig.8. Also, the dashboard shows the current running status of the machine as shown in the fig. 6 .

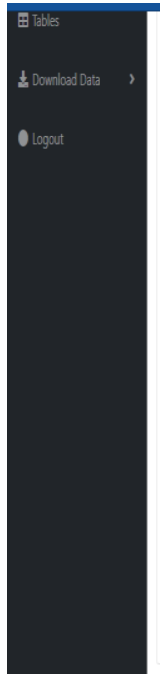

\begin{tabular}{|c|c|c|c|c|c|c|}
\hline TIME & 1. 10 & FREQUENCY & UNT(Thh) & VoltaGe & POWER_FACTOR & CURRENT \\
\hline $2019 \cdot 03-0202025520$ & 1 & 50.099001 & 38270 & 229.133316 & 0.907 & 0866738 \\
\hline $2019 \cdot 03-0202025634$ & 2 & 50.043999 & 38270 & 228.578537 & 0.912 & 083654 \\
\hline $2019 \cdot 03-120203019144$ & 3 & 50.089001 & 38300 & 227.125824 & 0.91 & 0.90258 \\
\hline $2019 \cdot 03-0203030650$ & 4 & 49976002 & 38320 & 227997095 & 0.908 & 09027 \\
\hline $2019-03-02003223553$ & 5 & 49.966 & 38390 & 230.225967 & 0.902 & 1.03914 \\
\hline $2019 \cdot 03 \cdot-02033.4532$ & 6 & 50.049 & 38.470 & 231.041504 & 0.906 & 125038 \\
\hline $2019 \cdot 03-0200355038$ & 7 & 50,400001 & 38490 & 230.599274 & 0.907 & 12452 \\
\hline $2019-03-0401: 5550$ & 8 & 50.063999 & 50.190 & 236.570999 & 0.886 & 02184 \\
\hline $2019 \cdot 03 \cdot 0401: 57: 45$ & 9 & 50.094002 & 50200 & 237.796814 & 0.884 & 021964 \\
\hline $2019 \cdot 03-040200916$ & 10 & 50.139 & 50210 & 239.211914 & 0.88 & 022476 \\
\hline Showing 1 to 10 of 1 & entries & & & Previd & 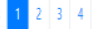 & ... 10 \\
\hline
\end{tabular}

Fig .5 Dashboard Table

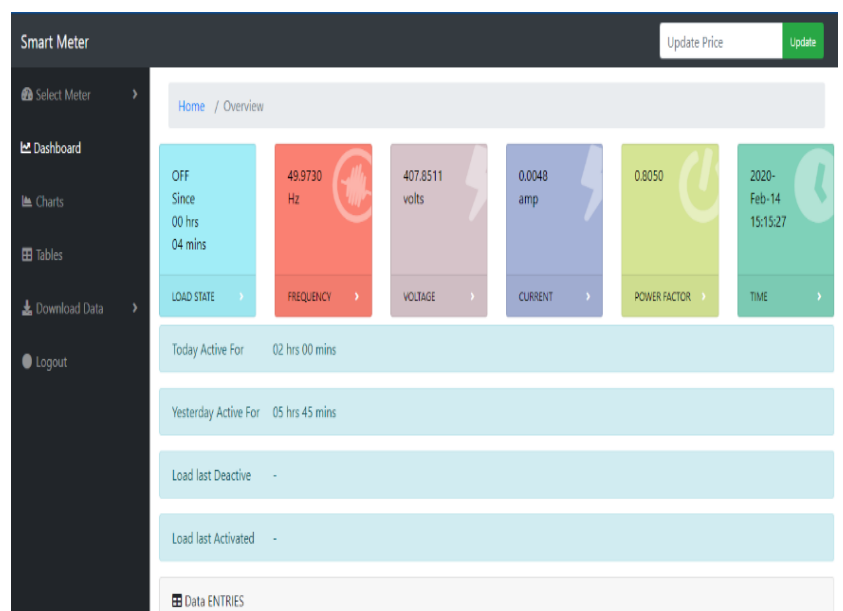

Fig. 6. Dashboard

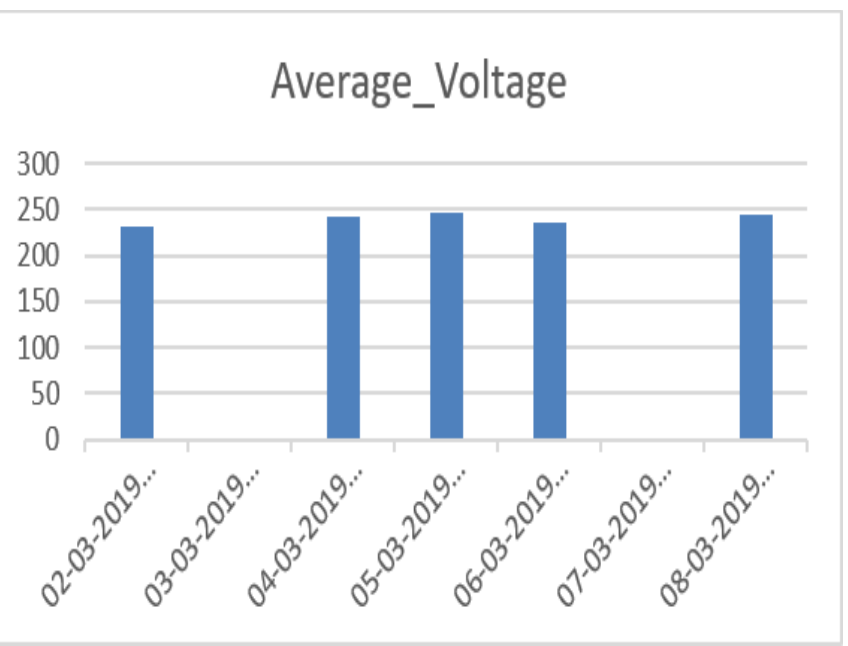

Fig. 7. Voltage consumption pattern

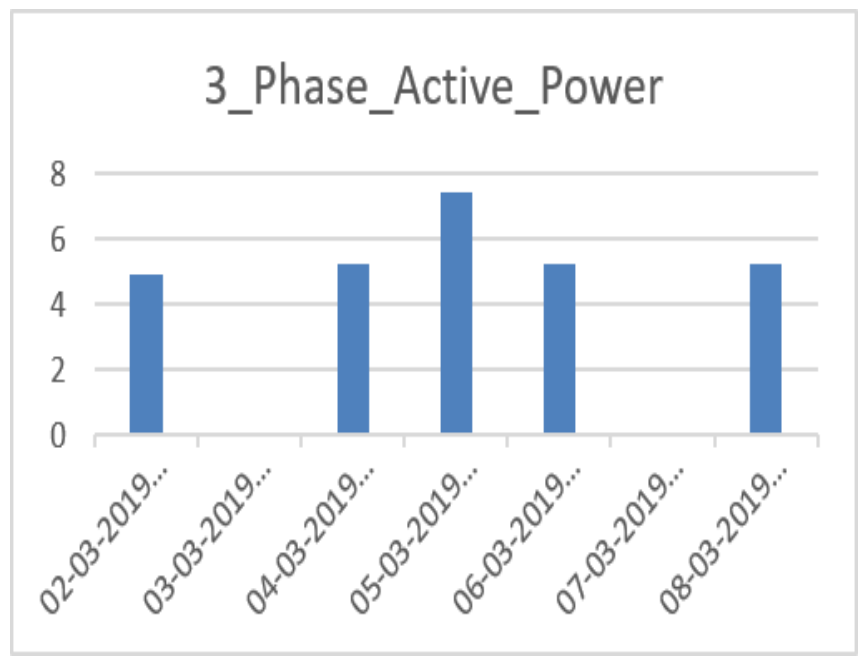

Fig.8. Energy Consumption pattern

\section{CONCLUSION\& FUTURE WORK}

The proposed system is based on the emerging technologies like IOT and cloud for real-time industrial energy meter monitoring and it provides the continuous meter data.

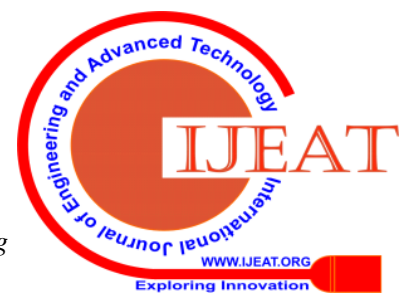




\section{Smart Industries in Industry4.0: An Iot and Cloud Based Real Time Energy Meter Monitoring System}

The importance of the proposed solution is to minimize man power requirement in monitoring the run time and down time and the quality of the power. An affordable prototype is designed to fetch, compute the real time meter data to an easy to use cloud dashboard.

Further, the developed work can be extended to apply the machine learning and data analytics on the collected data set to predict the future energy requirements of the plant and the predictive maintenance requirement. Also, the compressive sensing techniques and the advantage AI enabled hardware can be utilized to perform the edge analytics.

\section{REFERENCES}

1. https://kupdf.net/download/elite-440-modbusmapping_59faf59ee2b6f5bf6dec254e_pdf

2. https://www.raspberrypi.org/products/raspberry-pi-3-model-b-plus/

3. https://en.wikipedia.org/wiki/Modbus

4. http://www.securemeters.com/files/5714/7832/5529/Elite_440.pdf

5. https://www.electronics-notes.com/articles/connectivity/serial-datacommunications/rs485-introduction-basics.php

6. M. Jagadesh; M. Saravanan; V. Narayanan; M. Priya Vadhana ; K. Logeshwaran"Monitoring system in industrial iot"

7. https://www.researchgate.net/publication/330467093_Design_of_an_I oT_Energy_Monitoring_System

8. https://ieeexplore.iee.org/document/5752958

9. https://www.researchgate.net/publication/277141436_Automatic_Ene rgy_Meter_Reading_using_Smart_Energy_Meter

10. https://www.ijariit.com/manuscripts/v4i2/V4I2-1978.pdf

11. http://www.ijcea.com/wp-content/uploads/2018/04/80_CRC.pdf

12. https://irjet.net/archives/V5/i3/IRJET-V5I3612.pdf

\section{AUTHORS PROFILE}

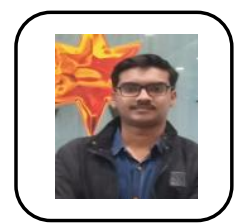

Prateek Agrawal is pursuing B.Tech in Electronics and Communication Engineering, Techno India NJR Institute of Technology, His area of interest is Embedded Software Developer.

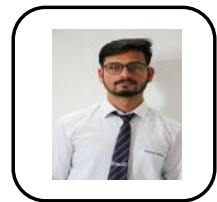

Himmat Khatik is a final year student pursuing B.Tech in Electronics and Communication Engineering Department, Techno India NJR Institute of Technology, His area of interest is IoT and embedded systems.

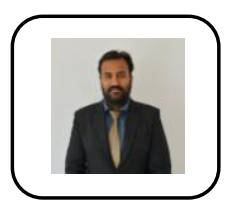

Yogendra Singh Solanki, is working as Asst Professor, Department of Electronics and Communications, Techno India NJR Institute of Technology, Udaipur. $\mathrm{He}$ is practicing the IoT Devices, AI/ML and VLSI in day to day life.

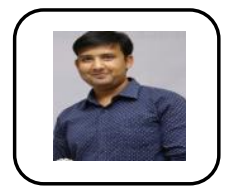

Aditya Maheshwari is working in the capacity of Project Lead- New Initiative \& Research at Techno India NJR Institute of Technology Udaipur. He is an Open Source Advocate, leading technical speaker and Expert of User Research (UI/UX). Leading students by creating and developing projects applying cloud technologies at an engineering institute. Achieved specialization and certification in IBM Watson and Blockchain, and applied in developing projects.

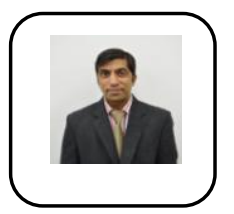

Dr. Vivek Jain, has received Ph.D. (Engg.) from MPUAT University 2017. He is working as assistant professor in Techno India NJR Institute of Technology. He has 9 publication. He has supervised one M.Tech. candidates successfully. His field of interest is DSP, IOT and VLSI. 